\title{
EDITORIAL
}

\section{New journal on biological frontiers}

\author{
Weihua WU \\ College of Biological Sciences, China Agricultural University, Beijing 100091, China
}

(C) Higher Education Press and Springer-Verlag Berlin Heidelberg 2010

Frontiers in Biology, an international peer-reviewed scientific journal to be published bi-monthly, is launched today. It is jointly published by Higher Education Press and Springer.

The journal provides a forum for a broad blend of peer-reviewed articles to promote rapid communication and exchanges among biologists around the world. The primary criterion for publication is new insights that are of broad interest to biologists, not just specialists, and the presentation of results must be appropriate to a wide audience of biologists. The journal will publish novel research of special significance in biology, especially in the areas of genomics, proteomics, neurobiology, genetics, developmental biology, biochemistry and molecular biology, and cell biology. We emphasize frontiers in the research areas in our scope which indicates either new scientific discoveries or exploration in technology.

The journal is an important forum and ideal vehicle for experimental reports in the most competitive and emerging areas. It will allow for rapid response to pre-submission inquiries, submission online or by e-mail, rapid review, special submission, and rapid publication on acceptance. We invite submissions of news, reviews, mini-reviews, research articles, and advanced techniques. The main topics will be bioinformatics, cell biology, developmental biology, molecular biology, molecular immunology, neurobiology, plant sciences, signal transduction, structural biology, and systems biology.

In the era of post genomics, the understanding of the life story has deepened from described biology to mechanism at molecules level even on a whole genome. "Hot" topics and the latest research into genomics (the entirety of an organism's hereditary information) and proteomics (the entire complement of proteins expressed by a genome, cell, tissue or organism) are probing the headspring of life functions. Biologists are increasingly studying through interaction network or cross talk either on genomics or proteomics controlling phenotypes to create an integrative biology. In addition, metabolization, the set of chemical reactions that occur in living organisms to maintain life, allows organisms to grow and reproduce, maintain their structures, and respond to their environments. Frontiers in metabolics are also revealing a unique world, which falls within our scope. In all living organisms, neurobiology encompases the most mysterious and greatest living actions, and biologists are increasingly making great efforts to understand the mysteries of neurobiology.

Frontiers in Biology will do its best to present the beauty of life and the life sciences to biologists.

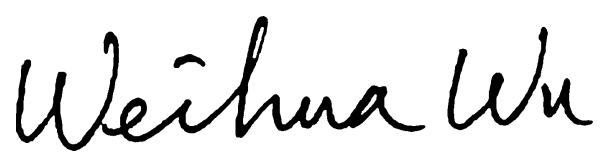

Weihua WU

Editor-in-Chief

Frontiers in Biology 